\title{
Preliminary evaluation on cerebral small vascular disease with cerebral arerio-venous pulse wave time
}

\author{
Yanxia Zhou, Pandeng Zhang, Xiang Tang, Rongkun Yang, Zhuqing Zhou, Chuming Huang, Yongti Xia
}

\begin{abstract}
In order to investigate the relationship between cerebral arerio-venous pulse wave time (CAV-PWT) and cerebral small vascular disease, this paper deals with 132 patients with cerebral small vascular disease and 115 sex-andage-matched control subjects. The pulse wave of terminal internal carotid artery and basal vein were simultaneously monitored by TCD(Transcranial Doppler), and the CAV-PWTs were calculated. The difference between the two groups' CAVPWT, and the relationships between CAV-PWT and demographic data and risk factors were analyzed. In the process of research, we got the following results. Firstly the normal range of CAV-PWT was $.1389 \pm .02429 \mathrm{~s}$ in normal people, while there were no significant statistical differences in the youth, middle-aged and elderly group. The CAV-PWT in middle-age group of lacunar cerebral infarction group and cerebral hemorrhage group, mixed group were significantly shortened than that in the control group, whereas there were no significant statistical differences in the elderly group. Secondly, the risk of cerebral arteriosclerosis with shortened CAV-PWT was 3.396 times than that of the normal people. And logistic regression analysis showed that age, CAV - PWT, hyperlipidemia, cerebral arteriosclerosis were more sensitive risk factors of lacunar cerebral infarction. $(\mathbf{P}<0.05)$. At last, the CAV-PWT in the moderate cerebral arteriosclerosis group were significantly shortened than that in the mild group. The results show that the CAV-PWT of patients with cerebral small vascular disease was shortened significantly, which might be associated with cerebral arteriolosclerosis.
\end{abstract}

Keywords-Cerebral small vascular disease; Pulse wave time; Transcranial Doppler; Arteriolosclerosis

\section{INTRODUCTION}

Cerebral small vessel disease (SVD) mainly manifests as the silent cerebrovascular disease, lacunar syndrome, vascular cognitive impairment and dementia, which is not easily recognized early. Cerebral arteriosclerosis is the most

Yanxia Zhou is with Department of Neurology, Shenzhen Second People's Hospital, Shenzhen, CO 518035 China (email: xiaxue159@163.com).

Pandeng Zhang is with Laboratory for Engineering and Scientific Computing, Shenzhen Institutes of Advanced Technology, Chinese Academy of Sciences; Shenzhen College of Advanced Technology, University of Chinese Academy of Sciences,Shenzhen, CO 518055 China (email:pd.zhang@ siat.ac.cn). Xiang Tang is with Department of Neurology, Shenzhen Second People's Hospital, Shenzhen, CO 518035 China.

Rongkun Yang is with Department of Neurology, Shenzhen Second People's Hospital, Shenzhen, CO 518035 China.

Zhuqing Zhou is with Department of Neurology, Shenzhen Second People's Hospital, Shenzhen, CO 518035 China.

Chuming Huang is with Department of Neurology, Shenzhen Second People's Hospital, Shenzhen, CO 518035 China.

Yongti Xia is with Department of Neurology, Shenzhen Second People's Hospital, Shenzhen, CO 518035 China. common cause of SVD ${ }^{[1]}$, and it is not well enough to detect or to quantify atherosclerosis situation with the current imaging techniques. With the pulse wave velocity (pulse wave velocity, PWV) detection technology is widely used in the cardiovascular field, in the methodological principle, it would be possible to observe elasticity of cerebral small artery. However, due to the difficulty to detect arterial distance between the two measuring points, especially the detection distance between intracranial vessels, unless invasive testing is applied, more errors could occur in simple surface detection, so more and more researches are inclined to use pulse transit time (pulse wave time, PWT) instead of PWV, to indirectly reflect the conduction velocity. In the past, transcranial Doppler (TCD) was also used to detect pulse wave time (PWT) between cerebral artery and vein, to evaluate the situation of cerebral arteriosclerosis in patients with lacunar infarction (LI) and showed significant shortening compared with the control group $^{[2]}$, which was similar in a lot of researches proving that patients with stroke had greater PWV ${ }^{[3]}$. However, as it is still in its infancy to detect CAV-PWT of intracranial arteries using TCD, it has not yet established normal reference data, and besides, neither is other methods to synchronize verification or bilateral contrast detection, nor is contrast detection grouped by age segment, which remains further improvement.

This study was to measure the normal range of CAVPWT in southern Chinese population, to compare CAVPWT between the normal control group and SVD groups, and to explore the correlation between CAV-PWT and each group of SVD, and to analyze age-adjusted subgroup of SVD, at the same time the findings of head MRA in the same period were compared, so as to understand whether it is an effective evaluation means of detecting cerebral arteriosclerosis using pulse transit time between cerebral artery and vein. Application of TCD in CAV-PWT of intracranial arteries, is a non-invasive, repeatable, and lowcost cerebrovascular detection technique, which is suitable for the community population with high risk factors to do the screening.

\section{SUBJECTS AND METHODS}

Subjects: case-control study method was applied. We enrolled patients with cerebral small vessel disease and the normal population from Department of Physical Examination from June 2014 to March 2015 in Shenzhen Second People's Hospital. According to imaging findings they were divided into the following groups: lacunar infarction group, mild white matter lesion group, CMBs group and mixed group; groups were also divided by age 
and sex, 20-39 years old as the youth group, 40-59 years old as the middle-aged group, $\geqslant 60$ years as the elderly group. The control group, according to cerebrovascular risk factors, was divided into 2 subgroups: completely normal control group and relatively normal control group with risk factors.

115 healthy volunteers were divided into two groups: one group with high risk factors $(n=34)$ (youth group $n=5$, middle-aged group $n=20$, elderly group $n=9)$, and the other group without risk factors $(n=81)$, (youth group $n=13$, middle-aged group $n=40$, elderly group $n=28$ ). For 132 patients in the case group were divided into the following four groups: 1) 70 in the pure lacunar infarction group (the middle-aged group $n=50$, the elderly group $n=20), 2$ ) 15 in the mild white matter lesion group (the middle-aged group $\mathrm{n}=8$, the elderly group $\mathrm{n}=7$ ), 3) 16 in cerebral microhemorrhage group (the middle-aged group $n=12$, elderly group $n=4$ ), and 4) the mixed group of 34 (the middle-aged group $\mathrm{n}=24$, the elderly group $\mathrm{n}=10$ ). The study was approved by the Ethics Committee of Shenzhen Second People's Hospital, and volunteers signed an informed consent.

Clinical data and routine examination: basic demographic information, registration of risk factors of cerebrovascular disease, imaging tests.

The instruments are provided uniformly and subjected to measurement certification. Field investigation and physical examination were done by formally trained and qualified physicians, and the data were summarized preserved by the research group. The study was approved by the ethics committee, after patients' informed consent.

Instruments and methods: Kai Tak 8100TCD instrument and two $1.6 \mathrm{MHz}$ intracranial probe were used. Detection process were carried out in the morning. Participants have a rest for 10 minutes, dual simultaneous monitoring of temporal windows are gathering on both sides of the BVR [3] and TICA, 55-70 $\mathrm{mm}$ in-depth inspection of BVR at bilateral temporal window was performed and in bilateral temporal window of about $83-84 \mathrm{~mm}$ in-depth inspection of TICA on the right. Pulse waves of two vessels were recorded and measured, when flow signals of both vessels are stable and envelope was complete and clear. Then calculate CAV-PWT between TICA and BVR on the right side. Each measurement was performed by two experienced physicians, and double-blind method was applied in the selection of satisfactory imaging and data measuring.

Measurement metrics: CAV-PWT measurement: time off point of pulse wave from bilateral TICA and bilateral BVR were measured in the same cardiac cycle and calculate the time difference between them as CAV-PWT. And 5 adjacent time differences were measured respectively, and the average of these five time differences was calculated as CAV-PWT. And at the same time peak velocity of blood flow velocity, average speed, PI and RI were recorded and averaged.

Statistical analysis: data analysis was performed using SPSS 19. Measurement data were tested by normality test. These measurement data in line with normal or near normal were showed as deviation, and the difference between the two groups was compared using $t$ test. These measurement data used median and interquartile for those which do not meet the normal distribution; rank test was used to compare the differences. Count data used $\chi^{2}$ test. The correlation between the multivariate used regression analysis or Logistic regression analysis. The level of significance for all statistical tests was set at $\mathrm{P}<0.05$.

\section{RESULTS}

\section{A.General data of subjects}

SVD groups were male-dominated, while the control group was dominated by women; The proportion of diabetes in the normal group with risk factors, lacunar infarction group and mixed group was greater than that in the normal group without risk factors, mild white matter lesion group and cerebral micro-hemorrhage group. The proportion of hypertension in the normal group with risk factors, lacunar infarction group, cerebral micro-hemorrhage group and mixed group was greater than that in the normal group without risk factors and mild white matter lesion group. There was no significant statistical difference between high cholesterol, age, smoking, alcohol consumption between each group. Fundus arteriosclerosis, carotid atherosclerosis and cerebral arteriosclerosis in the control group without risk factors showed significant difference compared with lacunar group and mixed group.

\section{B.The results of laboratory test}

Among these groups, head circumference, fasting glucose, cholesterol, LDL showed no statistically significant difference. The height of cerebral micro-hemorrhage group and mixed group was slightly higher than that of other groups, and the weight of normal group with risk factors and cerebral micro-hemorrhage was slightly greater than that of other groups, two hours postprandial blood sugar of normal group with risk factors, lacunar infarction group, cerebral micro-hemorrhage group and mixed group was higher than that of other groups; HCY level of mild white matter lesion group and cerebral micro-hemorrhage group was higher than that of other groups; HDL in normal group without risk factors and mixed group showed statistical difference; TG level of lacunar infarction group, cerebral micro-hemorrhage group and mixed group was high than that of other groups.

\section{C.Compare PWT time among age-adjusted groups}

Normal group: CAV-PWT was .1362 $\pm .03413 \mathrm{~s}$ in the youth group, and no statistical difference between the middle-aged group and elderly group (P>0.05). CAV-PWT of lacunar infarction group, cerebral micro-hemorrhage group and mixed group among the middle-aged participants was significantly shorter than that in the control group, which was of a statistical difference, but there was no significant statistical difference between the groups for the elderly, shown in table I.

Correlation analysis of CAV-PWT, demographic data and related risk factors: The dichotomy Multivariate Logistic regression analysis showed that: CAV-PWT .1389 $\pm .02429 \mathrm{~s}$ was set as normal; it showed no significant 
relevance between CAV-PWT and head circumference, height and weight; shortened CAV-PWT had no significant correlation with hypertension, diabetes, hyperlipidemia, carotid atherosclerosis and fundus arteriosclerosis, but the risk of patients with a shortened CAV-PWT to have cerebral atherosclerosis is 3.396 times as normal, shown in table II.

Logistic regression analysis by group was used to explore the correlation between lacunar infarction, mild white matter lesions and the risk factors related to cerebral micro-hemorrhage, conducting a case-control study. Sex, age, CAV-PWT, high cholesterol, diabetes, high blood pressure, fundus arteriosclerosis, cerebral arteriosclerosis, and carotid carotid atherosclerosis was considered as an independent variable, respectively; lacunar infarction, mild white matter lesions and cerebral microcirculation bleeding as the dependent variable for this group. Logistic regression analysis showed that age, CAV-PWT, hyperlipidemia, cerebral arteriosclerosis was more sensitive to the risk factors of lacunar infarction, $\mathrm{P}<0.05$; sex was a sensitive risk factor of mild white matter disease, OR value was 8.665 , $\mathrm{P}<0.05$; cerebral arteriosclerosis was a more sensitive risk factor of cerebral micro-hemorrhage, OR value was 0.192, $\mathrm{P}<0.05$. As shown in Table III.

\section{D.Cerebral arteriosclerosis are grouped according to head MRA examination}

50 cases with the mild cerebral arteriosclerosis and 20 cases the moderate cerebral arteriosclerosis. The CAV-PWT in the moderate cerebral arteriosclerosis group were significantly shortened than that in the mild group.

TABLE I. COMPARING OF PWT TIME AMONG AGE-ADJUSTED GROUPS

\begin{tabular}{|c|c|c|c|c|c|c|c|}
\hline Groups & Tcgwrf-65 & Tcgwrf-32 & LIg-70 & Mwmlg-15 & Cmg-16 & Mg-34 value \\
\hline $\mathrm{Mg}$ & $.1389 \pm .02429$ & $.1411 \pm .03114$ & $.1219 \pm .03834$ & $.1560 \pm .01386$ & $.1004 \pm 05622$ & $.1185 \pm .04183$ & $<0.05$ \\
\hline $\mathrm{Eg}$ & $.1378 \pm .04529$ & $.1610 \pm .01734$ & $.1362 \pm .03184$ & $.1629 \pm .03991$ & $.1298 \pm .02889$ & $.1350 \pm .03028$ & $>0.05$ \\
\hline
\end{tabular}

Tcgwrf-65: The control group without risk factors(n=65), Tcgwrf-32:The control group with risk factors(n=32), LIg-70:LI group(n=70), Mwmlg-15:Mild white matter lesion group(n=15), Cmg16:Cerebral micro-hemorrhage group( $\mathrm{n}=16)$, Mg-34:Mixed group( $\mathrm{n}=34), \mathrm{Mg}:$ Middle-aged group, Eg:Elderly group,

TABLE II. LOGISTIC REGRESSION ANALYSIS OF CAV-PWT AND HIGH RISK FACTORS OF STROKE

\begin{tabular}{|c|c|c|c|c|c|}
\hline Risk factors & B & Wals & P value & OR & 95\% C.I \\
\hline Hypertension & .296 & .417 & .518 & 1.344 & $.548-3.301$ \\
\hline Diabetes & -.472 & .534 & .465 & .624 & $.176-2.210$ \\
\hline Hyperlipidemia & -18.243 & .000 & .998 & .000 & .000 \\
\hline $\mathrm{Cta}$ & -.734 & .922 & .337 & .480 & $.107-2.148$ \\
\hline $\mathrm{Cba}$ & 1.223 & 7.157 & .007 & 3.396 & $1.387-8.318$ \\
\hline $\mathrm{Fa}$ & -.017 & .000 & .982 & .983 & $.212-4.564$ \\
\hline
\end{tabular}

TABLE III. LOGISTIC REGRESSION ANALYSIS OF LACUNAR INFARCTION AND DEMOGRAPHIC DATA AND RELATIVE RISK FACTORS

\begin{tabular}{|c|c|c|c|c|c|}
\hline Risk factors & B & Wals & P value & OR value & 95\% C.I \\
\hline Age & 1.788 & 11.696 & .001 & 5.977 & $2.145-16.655$ \\
\hline CAV-PWT & 1.724 & 3.976 & .046 & .178 & $.033-0.971$ \\
\hline Hyperlipidemia & 3.031 & 5.250 & 0.022 & 0.048 & $2.145-16.655$ \\
\hline Cba & 4.275 & 38.023 & .000 & 71.884 & $18.471-279.750$ \\
\hline
\end{tabular}

\section{DISCUSSION}

Cerebral small vessel disease mainly refers to a group of diseases involving small vessels with intracranial pathology; lesions include small arteries, arterioles, capillaries and venules, and their common causes are lipid hyaline degeneration, small vessel arteriosclerosis and atherosclerosis; other causes include cerebral amyloid angiopathy, cerebral autosomal dominant arteriopathy with subcortical infarcts and leukoencephalopathy, (CADASIL), vasculitis [4], and atherosclerosis of small vessels is the most common cause. Cerebral small blood vessels mainly involve small arteries from two vascular systems: superficial branches of leptomeningeal vessel and deep perforating arteries of large blood vessels. Both run towards the opposite direction, with these arteries called as "terminal arteries", which don't have arterial anastomosis, and form watershed area in the deep subcortical white matter. Acute 
lesions are prone to form lacunar infarction or bleeding, whereas chronic low perfusion relate to white matter damage. But now for the pathogenesis of cerebral small vessel disease, it has not yet been fully understood; it is presumed that cerebral small vessel disease was jointly caused by various risk factors for cerebrovascular disease and genetic factors, and its imaging performance of LI, loose white matter, and CMBs, alone or simultaneously. Despite recently some scholars believe that the pathogenesis of LI is closely related to hemodynamic abnormalities and micro-emboli, but most scholars still believe that it is the main cause that hypertension leads to microvascular lesions. It was reported in the literature that $47 \%-90 \%$ of LI with hypertension was associated with hyaline of tiny artery[5], namely small vessel arteriosclerosis. Cerebral microhemorrhage refers to the following imaging phenomenon in $\mathrm{T} 2 *$ weighted gradient echo sequence magnetic resonance imaging (GRE-T2*): small-lesion, circular and speckled low signal or signal missing, and peripheral edema[6].Imaging and pathology contrast studies suggest microbleed had close pathophysiologic relationship with cerebral small vessel disease [7], and with the severity of ischemic changes in white matter[8]. Ischemic demyelinating of white matter is an important symbol for cerebral small vessel disease to cause the damage of brain tissues; and it is called leukoaraiosis in neuroimaging, and process of white matter damage is an alternative endpoint in the evaluation of cerebral small vessel disease [9]. And hypertensive cerebral ischemia with white matter lesions showed more sclerosis of small arteries than those without white matter lesions, and the smaller the diameter of the small arteries, the higher the degree of sclerosis [10]. This study is to learn more about the degree of vascular atherosclerosis of the above three classic small vessel disease of the brain from the perspective of atherosclerosis.

Arteriosclerosis refers to changes ranging from vascular lumen to structure of the vascular wall. Currently numbers of imaging detection techniques are putting more emphasis on the detection of changes in the lumen of large vessels, and having poor detection results in small vessel lumen, and can't evaluate effectively physical properties, such as elasticity and compliance, caused by structural changes of vascular wall; so it is difficult to discern whether there is arteriosclerosis. Due to characteristics of structure, function and distribution of small arteries, in the presence of risk factors, decreased elasticity appeared earlier, which is the earliest manifestation of vascular disease ${ }^{[11]}$. Small artery elasticity can be screened to detect asymptomatic subclinical vascular disease, providing an objective basis for early aggressive intervention, so as to evaluate the effect of the intervention.

Arterial elasticity, also known as compliance, depends on the size of the artery lumen diameter and wall stiffness or expansion, and it is the inherent elastic properties of the arterial wall. When patients have arteriosclerosis or luminal stenosis and even occlusion, arterial elasticity change can occur. Nowadays, there are a lot of methods of evaluation of arterial elasticity, of which PWV is acknowledged as the more accurate method. However, due to relatively difficult to detect arterial distance between the two measuring points, in recent years the authors use noninvasive Doppler tissue imaging (DTI) technique to measure the aorta, PWT (measuring the abdominal aorta section from the aortic root to $2 \mathrm{~cm}$ below the diaphragm) to evaluate arterial elasticity, and results show that the more severe arteriosclerosis passed, the shorter the time, consistent with the theory of pulse wave velocity measurement ${ }^{[12]}$, which further confirms the practicality of pulse transit time measurement. Therefore, this study detected ipsilateral PWT between TICA and BVR by TCD, so that CAV-PWT can reflect arteriosclerosis situation of intracranial arteries. However, as CAV-PWT pulse wave is the conduction time, in addition to accurately reflect the elasticity and compliance of artery wall, the distance between the two measuring points, namely the conduction distance of pulse wave, also has a greater impact on its value. Previous studies and this study strongly suggest that CAV-PWT had no significant correlation with head circumference, indicating that the length difference of the small arteries from TICA the BVR had no effect on CAVPWT value.

Previous studies of PWV and PWT were concentrated more in the extracranial artery, and it is still at an initial stage for the study of micro-artery disease of the small artery and capillary segment between cerebral arteries and veins. For normal people, whether it is the youth group, middle-aged group or elderly group, CAV-PWT showed no significant statistical difference. But for the case group, due to very few cases in the youth group aged less than 40 years old, we only studied the youth group and elderly group. Patients with lacunar infarction, cerebral micro hemorrhage, or white matter lesions were divided into groups by age for further verification, and in the middle-aged group of patients from lacunar infarction group, cerebral microhemorrhage group and mixed group, CAV-PWT was significantly shorter than that in the control group, indicating the presence of sclerotic changes of arterioles and capillaries in cerebral small vessel disease. Correlation analysis of CAV-PWT and demographic data and risk factors also showed that shortened PWT was associated with cerebral arteriosclerosis, supporting the reliability of our results. Now there are researches on measuring circulation time of cerebral arteriovenous directly through ultrasound contrast agent [13], finding that patients with CADASIL, vascular dementia, Alzheimer's disease or stroke had significantly longer time than those in the control group. Because most of the time of cerebral circulation is the time passing from cerebral small arteries to capillaries, the extension of its value may also indirectly reflect the stiffness of small arteries and capillaries or occlusion changes. It is also proved by our study that the shortened CAV-PWT had the same meaning of measurement of cerebral venous circulation time, which is suggestive of the presence of cerebral small artery disease. However, compared with the simple detection of cerebral circulation time, PWV and CAV-PWT had higher accuracy and less error, because they are direct reflection of elasticity and compliance of vascular wall. furthermore, for CAV-PWT detection using TCD, it is simple, repeatable, and reduces the high-risk of application of ultrasound enhancer, so as to facilitate the screening of a 
large number relevant patients in the clinic.

However, the present study in elderly group and mild white matter lesions group did not draw statistically significant data. Considering the following two reasons, one is that the enrolled number of mild white matter lesions was less than that of other groups; the incidence of its hypertension, diabetes, hyperlipidemia, fundus arteriosclerosis, cerebral arteriosclerosis or carotid atherosclerosis was significantly lower than that of other groups, closer to the normal control group without risk factors. As is known to all, leukoaraiosis is a terminology of imaging diagnosis, seen in Alzheimer's disease (AD), subcortical arteriosclerotic encephalopathy, dementia, carbon monoxide poisoning, acquired immunodeficiency syndrome, and part of the healthy elderly. The occurrence of LA is closely related to circulation disorder of white matter adjacent to the lateral ventricle wall, cerebrospinal fluid circulation disorders and cerebral edema; temporarily no evidence showed that LA is directly related to atherosclerosis. But LA may result in subcortical infarction and cerebral hemorrhage, and is an independent predictor of stroke ${ }^{[14]}$; the more severe white matter lesions, the lower regional cerebral blood flow, the worse cerebral autoregulation $^{[15]}$, reflecting higher levels of ICAM-1, a blood marker of endothelial damage ${ }^{[16]}$. When LA with cerebral infarction, cerebral hemorrhage, cerebral micro hemorrhage is the mixed group of this study, representative of severe white matter lesions; So mixed group had more obvious correlation with cerebral arteriosclerosis, but it was not necessary that mild LA was related to cerebral arteriosclerosis. The other reason is that, the elderly group had less cases, due to bad unilateral or bilateral temporal window in most elderly patients which had adverse impact of FIG resolution; its statistics was for reference only, and it remains further verification by a larger sample. The results also showed PWT was significantly correlated with the degree of cerebral arteriosclerosis, but this elderly group mainly enrolled patients with mild or non-obvious cerebral arteriosclerosis, which affected the final results of the observation.

As we all know, advanced age, hypertension, diabetes or hyperlipidemia is a major risk factor for cardiovascular disease, the main reason for atherosclerosis, as well as a major contributing factor of atherosclerosis. In this study, group logistic regression analysis of risk factors of lacunar infarction, cerebral micro hemorrhage and white matter lesions, showed that age, CAV-PWT, hyperlipidemia and cerebral arteriosclerosis were relatively sensitive risk factors of lacunar infarction. Therefore, further proving that shortened CAV-PWT in lacunar infarction is one form of phenotype of cerebral arteriosclerosis, and is the major cause of arteriosclerosis leading to lacunar infarction(LI). Compared with carotid and fundus atherosclerosis, CAVPWT was more strongly associated with cerebral arteriosclerosis. But patients with LA and cerebral micro hemorrhage showed no significant correlation with PWT, hypertension, or diabetes, probably because of fewer cases recruited.

Numerous studies reveal that cerebral small vessel diseases may be an important risk factor of vascular dementia and cognitive decline. Preliminary studies have shown that routine use of TCD in detection of CAV-PWT, can detect cerebral arteriosclerosis at an early stage, and perform early intervention in asymptomatic patients with LI. Therefore, for patients cerebral small-vessel disease, in addition to actively control blood sugar, blood pressure, blood lipids, weight, regular TCD screening for stroke should be performed in high-risk groups; in addition to conventional monitoring of cerebral blood flow velocity, CAV-PWT is a reliable quantitative screening index reference. However, due to TCD in the detection of CAVPWT of intracranial arteries is still in its infancy, follow-up study of CAV-PWT remains blank, which is for further improvement.

\section{REFERENCES}

[1] Lammie, G. Alistair. "Pathology of small vessel stroke." British medical bulletin, vol.56 ,2000,pp. 296-306.

[2] Yanxia Zhou, Qingchun Gao, Yung-kun Yang, et al. " Cerebral lacunar infarction time between the initial evaluation of intravenous pulse wave." Chinese Journal of Nervous and Mental Diseases, vol.38, 2012, pp. 393-396(in Chinese).

[3] Nakano, Takahiro, Hiroki Ohkuma, and Shigeharu Suzuki. "Assessment of vascular injury in patients with stroke by measurement of pulse wave velocity." Journal of Stroke and Cerebrovascular Diseases, vol.13,2004, pp. 74-80.

[4] Arvanitakis, Zoe, et al. "Cerebral amyloid angiopathy pathology and cognitive domains in older persons." Annals of neurology, vol.69,2011, pp. 320-327.

[5] Sullivan, ColinE, et al. "Reversal of obstructive sleep apnoea by continuous positive airway pressure applied through the nares." The Lancet, vol.317,1981, pp. 862-865.

[6] Nighoghossian N, Hermier M, Adeleine P, et a1. "Old microbleeds are a potential risk factor for cerebral bleeding after ischemic stroke:a gradient-echo T2*-weighted brain MRI study." Stroke, vol. 33,2002, pp 735-742.

[7] Werring, D. J., et al. "Cerebral microbleeds are common in ischemic stroke but rare in TIA." Neurology, vol.65,2005, pp. 1914-1918.

[8] Kato H, Izumiyama M, Izumiyama $\mathrm{K}$, et a1. "Silent cerebral microbleeds on $\mathrm{T} 2 *$-weighted MRI:correlation with stroke subtype, stroke recurrence, and leukoaraiosis." Stroke,vol.33, 2002, pp. 15361540 .

[9] Sehmidt R, Seheltens P, Erkinjuntti T, et a1. "White matter lesion progression:a surrogate endpoint for trials in cerebral small-vessel disease." Neurology, vol. 63, 2004 , pp. 139-144.

[10] Yonghua Huang, Weiwei Zhang, Lang Lin et al. "Quantitative Leukoaraiosis small arteries with cerebral infarction patients with brain research." Chinese Journal of Geriatric Heart Brain Diseases,vol.10, 2008, pp.688-690(in Chinese).

[11] Grey, Elizabeth, et al. "Reduced small artery but not large artery elasticity is an independent risk marker for cardiovascular events." American journal of hypertension, vol.16, 2003, pp. 265-269.

[12] Lin Peng. "Research artery elasticity detection method." cardiovascular and cerebrovascular disease prevention and control,vol.7, 2007, pp.7-9(in Chinese).

[13] Liebetrau, Martin, et al. "Prolonged Cerebral Transit Time in CADASIL A Transcranial Ultrasound Study." Stroke, vol.33, 2002, pp. 509-512.

[14] Ross, Elliott D., et al. "Relationship of leukoaraiosis to cognitive decline and cognitive aging." Cognitive and behavioral neurology, vol.18, 2005, pp. 89-97.

[15] Fu, Jian Hui, et al. "Relationship between cerebral vasomotor reactivity and white matter lesions in elderly subjects without large artery occlusive disease." Journal of Neuroimaging, vol.16, 2006, pp. $120-125$.

[16] Han, Jing Hao, et al. "Plasma level of sICAM-1 is associated with the extent of white matter lesion among asymptomatic elderly subjects." Clinical neurology and neurosurgery, vol.111, 2009,pp. 847-851. 